CONCISE REPORT

\title{
Diagnosis and follow up of aortitis in the elderly
}

A K Scheel, J Meller, R Vosshenrich, E Kohlhoff, U Siefker, G A Müller, F Strutz

Ann Rheum Dis 2004;63:1507-1510. doi: 10.1136/ard.2003.015651

\begin{abstract}
Objectives: To evaluate the correlation of MRI and [ $\left.{ }^{18} \mathrm{~F}\right] \mathrm{FDG}-$ PET scans with the clinical course and inflammatory markers in patients with aortitis.

Methods: Eight patients with aortitis presenting with unspecific GCA-like symptoms were examined. Aortitis was diagnosed and followed up by [ ${ }^{18}$ F]FDG-PET and MRI. The aorta was divided into three vascular regions (ascending aorta, aortic arch, and descending aorta) to localise the aortic inflammation and compare both imaging techniques. Results were correlated with clinical and laboratory examinations.

Results: At diagnosis, 20/24 vascular regions from eight patients were positive by $\left[{ }^{18}\right.$ F]FDG-PET scan and $15 / 21$ aortic regions by MRI. Patients were treated with corticosteroids and followed up for a mean (SD) of 13.3 (4.7) months. In [ $\left.{ }^{18} \mathrm{~F}\right] \mathrm{FDG}-\mathrm{PET}, 11 / 20$ (55\%) initially pathological aortic regions returned to normal in the follow up examination, which correlated closely with the clinical and laboratory follow up examination. Conversely, in MRI, 14/15 initially affected vascular regions were unchanged.

Conclusions: $\left[{ }^{18} \mathrm{~F}\right]$ FDG-PET and MRI are both effective techniques for detecting early aortitis and have a high correlation with laboratory inflammatory measures. However, during the follow up examination, [ $\left.{ }^{18} \mathrm{~F}\right] \mathrm{FDG}-\mathrm{PET}$ uptake decreased in line with the clinical symptoms and inflammatory serum markers, whereas MRI scans gave more static results.
\end{abstract}

A ortitis may occur idiopathically ${ }^{1}$ as well as in Takayasu's arteritis (TA) and giant cell arteritis (GCA; temporal arteritis, polymyalgia rheumatica $(\mathrm{PMR}))$, in a number of rare rheumatological and infectious diseases, and in atherosclerosis. Most reports of aortitis describe TA and aortitis associated with GCA. GCA usually affects the medium sized extracranial arteries. ${ }^{2}$ However, the aorta may be affected in 10-15\% according to clinical criteria. ${ }^{3}$ Patients often present with non-specific clinical symptoms and raised serum markers of inflammation. These can be accompanied by more specific clinical symptoms evoked by arterial narrowing due to vessel wall inflammation (for example, decreased artery pulses, visual loss).

Traditionally, angiography has been used as the imaging "gold standard" for diagnosing vasculitis, detecting luminal abnormalities, but it is of limited use for detecting early mural findings. Magnetic resonance imaging (MRI) is less invasive, and may show vascular wall thickening early in the disease course when angiography is completely normal. ${ }^{4}$ First reports have described positron emission tomography with fluorine-18-fluorodeoxyglucose ( $\left[{ }^{18} \mathrm{~F}\right]$ FDG-PET) as a possible sensitive tool in diagnosing aortitis in patients with GCA. ${ }^{5-8}$

Because GCA is often not diagnosed until stenosis occurs, new diagnostic tools are needed for early diagnosis. PET and MRI are the most promising for this purpose. In our previous study, ${ }^{8}$ not all aortic regions could be examined with both imaging techniques and no clinical data were available. We therefore now describe the first data of an elderly patient cohort presenting with non-specific signs of inflammation caused by aortitis at two times, directly comparing aortic regions with $\left[{ }^{18} \mathrm{~F}\right] \mathrm{FDG}$-PET and MRI at follow up examinations.

\section{PATIENTS AND METHODS}

Patients and clinical examination (CE)

Eight consecutive patients (6 female, 2 male, age 57-76, median 63 years) were diagnosed with an undifferentiated aortitis. All patients underwent CE, MRI, and PET twice (mean (SD) interval 13.3 (4.7) months, median 5.5). Routine laboratory measures were assessed in all patients at both visits, and HLA typing was performed.

Four of eight patients fulfilled the GCA criteria by age at onset, newly diagnosed headache, and highly raised erythrocyte sedimentation rate (ESR). ${ }^{\circ}$ Doppler ultrasound examinations of the temporal ${ }^{10}$ and carotid arteries were unremarkable in five patients. Three patients had PMR. ${ }^{11}$ Patients only showed minor clinical symptoms evoked by aortic vessel wall inflammation: two patients each presented with arm claudication and showed a significant difference in systolic blood pressure $(>20 \mathrm{~mm} \mathrm{Hg}$ ) between both arms. However, no bruits could be detected clinically. Although unspecific, seven patients had arterial hypertension, indicating possible renal artery involvement. Before diagnosis, patients presented with non-specific symptoms (exhaustion $(n=5)$, weight loss $(n=5)$, night sweats $(n=5)$, headache $(n=4)$, fever of unknown aetiology $(n=3))$. A CE disclosed no inflammatory signs of temporal and carotid arteries. Patients did not exhibit jaw claudication, visual problems, or signs of infection. Biopsy of the temporal arteries was not performed because involvement was deemed unlikely by clinical criteria.

\section{Imaging techniques \\ MRI}

MRI was performed with 1.5 T MRI systems (MagnetomVision and Symphony/Quantum, Siemens, Germany). In MRI, aortic wall thickening $(\Delta 2 \mathrm{~mm})$ and contrast agent enhancement of the vessel wall on transversal MR images was indicative of early aortitis. MRI datasets were evaluated and scored independently by two readers.

\section{$\left[{ }^{18} \mathrm{~F}\right] \mathrm{FDG}-\mathrm{PET}$ \\ $\left[{ }^{18}\right.$ F]FDG-PET was performed with a hybrid camera (hPET; DHCC, Prism2000XP, Marconi Medical Systems, Germany) in four patients at baseline or with a dedicated system (dPET; ECAT HRplus, Siemens, USA) in four patients at baseline and}

\footnotetext{
Abbreviations: $\mathrm{CE}$, clinical examination; $\mathrm{CRP}, \mathrm{C}$ reactive protein; $\mathrm{ESR}$, erythrocyte sedimentation rate; $\left[{ }^{18} \mathrm{~F}\right] \mathrm{FDG}-\mathrm{PET}$, positron emission tomography with fluorine-18-fluorodeoxyglucose; GCA, giant cell arteritis; PMR, polymyalgia rheumatica TA, Takayasu's arteritis; VR, vascular regions
} 

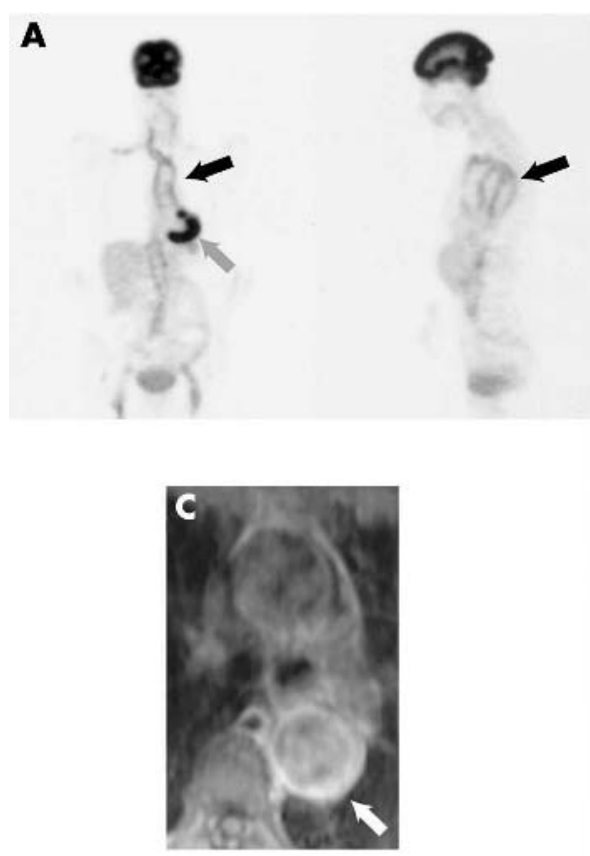

B
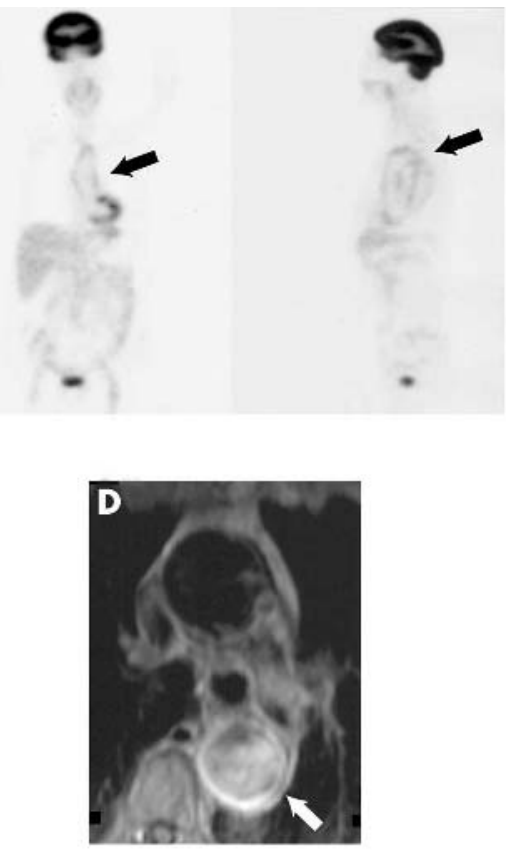

Figure 1 PET and MRI images of a female patient with aortitis (64 years) at the time of diagnosis ( $A$ and $C$ ) and 3 months later (B and D). (A) and (B) display PET images of a 64 year old patient. Compared with liver tissue, an uptake can be visualised at the ascending aorta, aortic arch, descending (black arrows) and abdominal aorta (A). Three months later there is still an uptake at the ascending aorta aortic arch and descending aorta (arrow). However, comparing the uptake with liver tissue, we see an uptake of equal intensity, interpreted as improvement of the aortic inflammation (B). (C) and (D) show MRI images of the same patient with contrast agent enhancement of the aortic wall (descending aorta) of equal intensity (white arrows) at both times indicating the more "static" results obtained with that imaging technique. The grey arrow in A depicts unspecific staining of the heart.

eight patients at the follow up visit. Transaxial tomograms of our patient and reference group were evaluated and scored by two independent readers who were unaware of the MRI results. In PET, semiquantitative analysis was performed by comparing uptake in the large vessels with uptake in the liver tissue. ${ }^{8}$

Seven patients underwent PET and MRI twice. One patient underwent PET twice and could not be examined with MRI owing to a cardiac pacemaker. For point to point comparison of both imaging techniques, we divided the aorta into three vascular regions (VR): ascending aorta, aortic arch, and descending aorta. We then compared the imaging data with clinical and laboratory findings and correlated PET with MRI using a scoring system indicating " $\mathrm{l}$ " for "inflammation" and " 0 " for "no inflammation", with limits of positivity as described before. ${ }^{8}$

Comparisons of follow up laboratory measures were performed with a paired $t$ test. Correlation coefficients for MRI and PET were calculated according to Spearman. Critical value tables for Spearman correlation were used to determine correlation coefficients. HLA typing correlations were determined by the binomial test.

\section{RESULTS}

\section{Clinical and laboratory measures}

After diagnosis, all patients were treated with immunosuppressive drugs (eight high dose corticosteroids; four, additionally, methotrexate). At baseline, all patients had raised serum markers of inflammation (mean (SD) C reactive protein (CRP) 77.9 (8.7) mg/l, ESR 80.8 (8.0) mm/lst h, fibrinogen $8.5(9.5) \mathrm{g} / \mathrm{l})$. With treatment, all patients experienced robust clinical improvement (exhaustion $(\mathrm{n}=2)$, weight loss $(n=0)$, night sweats $(n=1)$, headache $(n=0)$, fever of unknown aetiology $(\mathrm{n}=0))$ and a marked reduction of serum markers of inflammation (mean CRP 5.1 (1.5) mg/l,
ESR 15.9 (3.5) mm/lst h, fibrinogen 3.8 (3.9) g/l; differences CRP and ESR: $p \leqslant 0.001$, fibrinogen: $p=0.003)$. Before treatment, patients exhibited normochromic anaemia (haemoglobin $108.9(8.2) \mathrm{g} / \mathrm{l}$ ) and reactive thrombocytosis $\left(508(45) \times 10^{9} / 1\right)$, which normalised during the follow up examination (haemoglobin 142.1 (3.8) g/l, platelets 318 $(18) \times 10^{9} / \mathrm{l}$; difference between visits $\mathrm{p}=0.004, \mathrm{p}=0.006$, respectively). Conversely, leucocyte counts were normal at both times. Four of eight patients were associated with HLADR4, which was-although the patient cohort was smallsignificant compared with a healthy population (13.3\%) $(\mathrm{p}=0.028) .{ }^{12}$

\section{Imaging data and comparison with laboratory variables}

Initially, all eight patients showed pathological uptake of the aorta in $\left[{ }^{18} \mathrm{~F}\right]$ FDG-PET (20/24 VR): six ascending aorta, six aortic arch, eight descending aorta. At baseline, MRI detected aortic inflammation in 15/21 VR (three ascending aorta, five aortic arch, seven descending aorta). In $\left[{ }^{18}\right.$ F]FDG-PET, 11/20 $(55 \%)$ initially pathological aortic VR returned to normal during a follow up examination, which correlated with clinical and laboratory examinations (CRP: $r_{\mathrm{s}}=0.645$, $\mathrm{p}=0.007 ;$ ESR: $r_{\mathrm{s}}=0.773, \mathrm{p} \leqslant 0.001$; fibrinogen: $r_{\mathrm{s}}=0.798$ ， $\mathrm{p} \leqslant 0.001)$. Besides aortic regions, $\left[{ }^{18} \mathrm{~F}\right] \mathrm{FDG}-\mathrm{PET}$ showed uptake in subclavian $(n=3)$, carotid $(n=5)$, and iliac arteries $(n=5)$ at the time of diagnosis. During follow up, uptake was detected in the subclavian artery $(n=0)$, whereas little uptake was seen in the carotid $(n=1)$ and iliac arteries $(\mathrm{n}=1)$.

Follow up examination of MRI gave more static results than PET (fig 1). Of 15 initially affected VR, 14 were unchanged and aortic inflammation improved in only one. There were no correlations of MRI follow up results with serum markers of inflammation (CRP: $r_{\mathrm{s}}=-0.117$, 
Table 1 (A) PET data $(n=8)$ at baseline and follow up; (B) MRI data $(n=7)$ at baseline and follow up

\begin{tabular}{|c|c|c|c|c|c|c|c|c|}
\hline \multirow[b]{2}{*}{ Patient } & \multirow[b]{2}{*}{ Sex } & \multirow[b]{2}{*}{$\begin{array}{l}\text { Age at } \\
\text { onset }\end{array}$} & \multicolumn{3}{|l|}{ Baseline } & \multicolumn{3}{|c|}{ Follow up } \\
\hline & & & $\begin{array}{l}\text { Ascend. } \\
\text { aorta }\end{array}$ & $\begin{array}{l}\text { Aortic } \\
\text { arch }\end{array}$ & $\begin{array}{l}\text { Descend. } \\
\text { aorta }\end{array}$ & $\begin{array}{l}\text { Ascend. } \\
\text { aorta }\end{array}$ & $\begin{array}{l}\text { Aortic } \\
\text { arch }\end{array}$ & $\begin{array}{l}\text { Descend. } \\
\text { aorta }\end{array}$ \\
\hline \multicolumn{9}{|c|}{ (A) PET data } \\
\hline 1 & $\mathrm{~F}$ & 60 & 1 & 0 & 1 & 0 & 1 & 1 \\
\hline 2 & $\mathrm{~F}$ & 63 & 1 & 1 & 1 & 0 & 1 & 1 \\
\hline 3 & $M$ & 64 & 1 & 1 & 1 & 0 & 0 & 1 \\
\hline 4 & $\mathrm{~F}$ & 76 & 0 & 1 & 1 & 0 & 0 & 1 \\
\hline 5 & $\mathrm{~F}$ & 57 & 0 & 0 & 1 & 0 & 0 & 1 \\
\hline 6 & $\mathrm{~F}$ & 63 & 1 & 1 & 1 & 0 & 0 & 1 \\
\hline 7 & $\mathrm{~F}$ & 62 & 1 & 1 & 1 & 0 & 1 & 0 \\
\hline 8 & $M$ & 66 & 1 & 1 & 1 & 0 & 1 & 0 \\
\hline \multicolumn{9}{|c|}{ (B) MRI data } \\
\hline 1 & $\mathrm{~F}$ & 60 & 0 & 1 & 1 & 0 & 1 & 1 \\
\hline 2 & $\mathrm{~F}$ & 63 & 1 & 1 & 1 & 1 & 1 & 1 \\
\hline 3 & $M$ & 64 & 1 & 1 & 1 & 1 & 1 & 1 \\
\hline 4 & $\mathrm{~F}$ & 76 & 1 & 1 & 1 & 1 & 1 & 1 \\
\hline 5 & $\mathrm{~F}$ & 57 & - & - & - & - & - & - \\
\hline 6 & $\mathrm{~F}$ & 63 & 0 & 0 & 1 & 0 & 0 & 0 \\
\hline 7 & $\mathrm{~F}$ & 62 & 0 & 1 & 1 & 0 & 1 & 1 \\
\hline 8 & $M$ & 66 & 0 & 0 & 1 & 0 & 0 & 1 \\
\hline
\end{tabular}

$\mathrm{p}=0.691 ; \quad$ ESR: $r_{\mathrm{s}}=-0.337, \mathrm{p}=0.260 ;$ fibrinogen: $r_{\mathrm{s}}=$ $-0.204, p=0.504)$. Table 1 presents detailed information about inflamed VR at baseline and the follow up visit.

Methotrexate had no additional effect on the outcome of either the MRI or the PET results. However, it was possible to reduce glucocorticoid dosage (mean prednisolone in the methotrexate group $5 \mathrm{mg}$, without methotrexate $9 \mathrm{mg}$ ).

Concordance in evaluation of imaging data could be obtained in $90 \%$ and $92 \%$ of the examined VR for MRI and PET, respectively.

\section{DISCUSSION}

Our data indicate that $\left[{ }^{18} \mathrm{~F}\right] \mathrm{FDG}$-PET is highly effective in detecting inflammatory aortic changes, with a good correlation with serum markers of inflammation. $\left[{ }^{18} \mathrm{~F}\right] \mathrm{FDG}-\mathrm{PET}$ was reliable for screening, but MRI was as effective in diagnosing disease within the aortic wall. However, when both imaging modalities were compared in a follow up examination, $\left[{ }^{18} \mathrm{~F}\right] \mathrm{FDG}$-PET was more reliable than MRI in detecting aortitis in correlation with clinical and laboratory measures. MRI showed more static aortic inflammation and a poor correlation with laboratory inflammatory markers. Interestingly, similar findings have been described with MRI in patients with $\mathrm{TA}^{13}$ confirming the apparent limitations of MRI in a follow up examination of vessel inflammation. Thus, $\left[{ }^{18} \mathrm{~F}\right]$ FDG-PET may be better than MRI in follow up analysis of aortitis; these results require confirmation in larger clinical trials. Alternatively, persistence of contrast media enhancement in MRI scans may indicate subclinical inflammation not detected by laboratory examinations and $\left[{ }^{18}\right.$ F]FDG-PET. Glucocorticoids are known to decrease the translocation of the intracellular GLUT-1 pool to the cell membrane and may alter the transmembranous glucose transport. PET diagnosis is based on glucose metabolism. Therefore, a decrease may be a reason for the close correlation with the decrease of inflammation after glucocorticoid treatment. However, this explanation is rather unlikely given the close correlation of the PET results with inflammatory markers.

Diagnosing aortitis is clinically challenging. Our patients presented with non-specific symptoms of inflammation, and an infection was excluded. Four patients could clinically be classified as GCA and three as PMR. Temporal artery biopsy was not performed because they were clinically unremarkable. Duhot and coworkers compared the clinical findings in biopsy positive and biopsy negative cases of patients with GCA. ${ }^{14}$ They found that blindness and jaw claudication were more common in biopsy positive cases, whereas headache and PMR were more common in biopsy negative cases, the latter closely representing our patient group. However, without biopsy, TA cannot be completely ruled out. Owing to typical clinical and laboratory findings and a good response to glucocorticoids, we deem GCA to be the most likely cause of aortitis. A major differential diagnosis from vasculitis in $\left[{ }^{18} \mathrm{~F}\right]$ FDG-PET uptake is atherosclerosis. ${ }^{15}$ However, intense vascular $\left[{ }^{18} \mathrm{~F}\right] \mathrm{FDG}$ uptake as seen in our patients in combination with the topography of the vascular involvement strongly suggests a diagnosis of vasculitis as compared with the moderate uptake in atherosclerosis. ${ }^{15}$

The selection of the patients in this retrospective study was based on clinical findings, and all patients had positive findings in $\left[{ }^{18} \mathrm{~F}\right]$ FDG-PET confirmed by MRI. A larger prospective follow up study will evaluate sensitivities and specificities of both methods.

In summary, we propose that clinicians should suspect aortitis in patients with non-specific signs of inflammation. With $\left[{ }^{18} \mathrm{~F}\right]$ FDG-PET, aortitis may be detected more often and clinicians need to be alert to its diagnosis. We found a good correlation in diagnosing aortitis with the initial $\left[{ }^{18} \mathrm{~F}\right] \mathrm{FDG}$ PET and MRI examinations, but during follow up MRI showed more static results.

\section{Authors' affiliations}

A K Scheel, E Kohlhoff, G A Müller, F Strutz, Department of Medicine, Nephrology and Rheumatology, Georg-August-University Göttingen, Robert-Koch-Strasse 40, D-37075 Göttingen, Germany

J Meller, U Siefker, Department of Nuclear Medicine, Georg-AugustUniversity Göttingen, Robert-Koch-Strasse 40, D-37075 Göttingen, Germany

R Vosshenrich, Department of Radiology, Georg-August-University Göttingen, Robert-Koch-Strasse 40, D-37075 Göttingen, Germany

Correspondence to: Dr A K Scheel; ascheel@gwdg.de

Accepted 27 January 2004 


\section{REFERENCES}

1 Parums DV, Dunn DC, Dixon AK, Mitchinson MJ. Characterization of inflammatory cells in a patient with chronic periaortitis. Am J Cardiovasc Pathol 1990;3:121-9.

2 Salvarani C, Cantini F, Boiardi L, Hunder GG. Polymyalgia rheumatica and giant cell arteritis. N Engl J Med 2002;347:261-71.

3 Klein RG, Hunder GG, Stanson AW, Sheps SG. Large artery involvement in giant cell (temporal) arteritis. Ann Intern Med 1975;83:806-12.

4 Matsunaga N, Hayashi K, Sakamoto I, Matsuoka Y, Ogawa Y, Honjo K, et al. Takayasu arteritis: MR manifestations and diagnosis of acute and chronic phase. Magn Reson Imaging 1998;8:406-14.

5 Blockmanns D, Maes A, Stroobants S, Nuyts J, Bormans G, Knockaert D, et al. New arguments for a vasculitic nature of polymyalgia rheumatica using positron emission tomography. Rheumatology (Oxford) 1999:38:444-7.

6 Blockmans D, Stroobants S, Maes A, Mortelmans L. Positron emission tomography in giant cell arteritis and polymyalgia rheumatica: evidence for inflammation of the aortic arch. Am J Med 2000;108:246-9.

7 Meller J, Grabbe E, Becker W, Vosshenrich R. Value of F-18-FDG-hybrid camera PET and MRI in early Takayasu aortitis. Eur Radiol 2003;13:400-5.

8 Meller J, Strutz F, Siefker U, Scheel AK, Sahlmann CO, Lehmann K, et al. Early diagnosis and follow-up of aortitis with [18F]FDG-PET and MRI. Eur J Nucl Med Mol Imaging 2003;30:730-6.
9 Hunder GG, Bloch DA, Michel BA, Stevens MB, Arend WP, Calabrese LH, et al. 1990 criteria for the classification of giant cell arteritis. Arthritis Rheum 1990;33:1122-8

10 Schmidt WA, Kraft HE, Vorpahl K, Volker L, Gromnica-Ihle EJ. Color duplex ultrasonography in the diagnosis of temporal arteritis. N Engl J Med 1997; 337: 1336-42.

11 Bird HA, Esselinckx W, Dixin AS, Mowat AG, Wood PH. An evaluation of criteria for polymyalgia rheumatica. Ann Rheum Dis 1979:38:434-9.

12 Müller CR, Ehninger G, Goldmann SF. Gene and haplotype frequencies for the loci HLA-A, HLA-B, and HLA-DR based over 13,000 German blood donors. Hum Immunol 2003;64:137-51.

13 Tso E, Flamm SD, White RD, Schvartzman PR, Mascha E, Hoffmenn GS Takayasu arteritis. Utility and limitations of magnetic resonance imaging in diagnosis and treatment. Arthritis Rheum 2002;46:1634-42.

14 Duhot $P$, Pinede L, Bornet $H$, Delomombe-Ragué S, Dumontet $C$, Ninet J, et al. Biopsy proven and biopsy negative temporal arteritis: differences in clinical spectrum at the onset of the disease. Groupe de recherche sur larterite a cellules geantes. Ann Rheum Dis 1999;58:335-41.

15 Belhocine T, Blockmans D, Hustinx R, Vandevivere J, Mortelmans L. Imaging of large vessel vasculitis with ${ }^{18} \mathrm{FDG}$-PET: illusion or reality? A critical review of the literature data. Eur J Nucl Med Mol Imaging 2003;30:1305-13. 